\title{
In Vitro Antifungal Activity of Cysteine Derivatives and their Combinations with Antifungal Agents Against Clinically Relevant Scedosporium species
} László Galgóczy ${ }^{1,2,}$, Mónika Homa ${ }^{1}$, Tamás Papp ${ }^{1}$, Palanisamy Manikandan ${ }^{3,4}$ and Csaba Vágvölgyi ${ }^{1}$

${ }^{1}$ University of Szeged, Faculty of Science and Informatics, Department of Microbiology, Közép fasor 52, H-6726 Szeged, Hungary

${ }^{2}$ Medical University of Innsbruck, Biocenter, Division of Molecular Biology, Innrain 80-82, A-6020, Innsbruck, Austria

${ }^{3}$ Department of Microbiology and Molecular Biology, Aravind Eye Hospital and Postgraduate Institute of Ophthalmology, Coimbatore-641014, Tamilnadu, India

${ }^{4}$ College of Applied Medical Sciences, Majmaah University, Majmaah 11952, Kingdom of Saudi Arabia

\begin{abstract}
Background: Members of the genus Scedosporium are emerging human pathogens, causing a wide range of infections. Their treatment means a challenge for clinicians, mostly because of the low susceptibility of these causative agents to conventional antifungal drugs. New alternative therapeutic approaches are required for better clinical outcomes. Cysteine and its derivatives alone or in combinations with antifungal drugs might have improved antifungal effects against Scedosporium species.

Methods: The in vitro antifungal effects of two cysteine forms and three of their derivatives (i.e., D-cysteine, L-cysteine, L-cysteine-methyl ester hydrochloride, N-isobutyryl-D-cysteine, and $\mathrm{N}$-isobutyryl-L-cysteine) were determined using the CLSI M38-A2 broth microdilution method. The in vitro drug interactions between the most effective cysteine derivatives and some conventional antifungal drugs were also investigated using checkerboard assays in case of four clinical isolates.

Results: L-cysteine-methyl ester hydrochloride proved to be the most effective among the investigated compounds. N-isobutyryl-L-cysteine exhibited antifungal activity against one Scedosporium aurantiacum strain only. D-cysteine, L-cysteine and $\mathrm{N}$-isobutyryl-D-cysteine proved to be ineffective against Scedosporium spp. in the investigated concentration range. No significant differences were found in the susceptibilities between environmental and clinical isolates. L-cysteine-methyl ester hydrochloride could interact synergistically with amphotericin B, caspofungin, terbinafine and voriconazole. Antagonistic interactions were not observed between the investigated compounds.

Conclusions: The present study provides an evidence for the in vitro antifungal activity of L-cysteinemethyl ester hydrochloride and the synergistic interactions between this cysteine derivative and conventional antifungal agents. Based on our results, L-cysteine-methyl ester hydrochloride in combination with antifungal drugs could be applicable in the treatment of human Scedosporium infections in the future. However, further studies are required to clarify the antifungal mechanism of L-cysteine-methyl ester hydrochloride and to investigate its activity in vivo.
\end{abstract}

\section{Introduction}

Scedosporium species are emerging human pathogens, responsible for a broad spectrum of infections, including superficial or deep localized diseases in immunocompetent hosts, and disseminated infections in immunocompromised or in near-drowning patients [1]. Scedosporium boydii and Scedosporium apiospermium are mainly associated with infections in temperate climates (e.g., Central Europe), while Scedosporium aurantiacum and Scedosporium prolificans infections are predominant in hot and arid countries (e.g., Spain, Australia) [2]. According to a recent clinical study in Spain, the members of this genus are the second most frequently isolated filamentous fungi from human infections after Aspergillus species [3]. The clinical manifestations of Scedosporium infections are very similar to those of aspergillosis, fusariosis and other hyalohyphomycosis [4]. The proper diagnosis is complicated by the low interspecies diversity and high intraspecies variability, especially within the S. boydii species complex. However, the accurate identification of the causative agent is crucial to find the most effective therapeutic approach, since the antifungal susceptibility profile of Scedosporium species is different from those of hyaline filamentous fungi, and also varies within the genus itself [2]. Another challenge in the treatment is the frequently observed antifungal resistance of the Scedosporium isolates to conventional antifungal agents [1]. In a consequence of these, clinicians have limited options to treat Scedosporium

\section{Publication History:}

Received: June 25, 2016

Accepted: August 09, 2016

Published: August 11, 2016

\section{Keywords:}

In vitro, Cysteine derivatives, Antifungala activity, Scedosporium infections. Therefore, new therapeutic strategies are required beside of the currently available ones.

Cysteine and their derivatives have been previously reported to have antifungal activity against different filamentous fungi (Table 1) [5-11]. Previously we proved the antifungal activity of N-acetyl-L-cysteine against Scedosporium species [12]. D-cysteine (DC), L-cysteine (LC), L-cysteine-methyl ester (LCME), N-isobutyryl-D-cysteine (NIDC), and N-isobutyryl-L-cysteine (NILC) showed antifungal effect against species belonging to Mucorales [6]. LC was reported to inhibit spore germination and to cause reduced hyphal growth in different filamentous fungi (e.g. dermatohytes, Aspergillus spp., Fusarium spp.) $[7,8,11]$.

The aims of the present study were (i) to determine the in vitro susceptibility of clinical and environmental Scedosporium isolates "Corresponding Author: Dr. László Galgóczy, Faculty of Science and Informatics, Department of Microbiology, Közép fasor 52, H-6726 Szeged, Hungary, Tel: +36 62 544005; E-mail: galgoczi@gmail.com

Citation: Galgóczy L, Homa M, Papp T, Manikandan P, Vágvölgyi C (2016) In Vitro Antifungal Activity of Cysteine Derivatives and their Combinations with Antifungal Agents Against Clinically Relevant Scedosporium species. Int J Clin Med Microbiol 1: 111. doi: https://doi.org/10.15344/2456-4028/2016/111

Copyright: (C) 2016 Galgóczy et al. This is an open-access article distributed under the terms of the Creative Commons Attribution License, which permits unrestricted use, distribution, and reproduction in any medium, provided the original author and source are credited. 
Citation: Galgóczy L, Homa M, Papp T, Manikandan P, Vágvölgyi C (2016) In Vitro Antifungal Activity of Cysteine Derivatives and their Combinations with Antifungal Agents Against Clinically Relevant Scedosporium species. Int J Clin Med Microbiol 1: 111. doi: https://doi.org/10.15344/2456-4028/2016/111

Page 2 of 5

\begin{tabular}{|c|c|c|c|c|}
\hline $\begin{array}{l}\text { Cysteine/ } \\
\text { derivatives }^{a}\end{array}$ & Chemical structure $^{b}$ & Investigated organisms & Antifungal effect/mode of action & References \\
\hline \multirow{2}{*}{ DC } & & Eutypa lata & $50 \%$ growth inhibition & [5] \\
\hline & & Mucorales spp. & Reduced hyphal growth, MEC: $0.625-10 \mathrm{mM}$ & {$[6]$} \\
\hline \multirow{6}{*}{$\mathrm{LC}$} & \multirow{6}{*}{$\mathrm{HS}$} & Dermatohytes & $\begin{array}{c}\text { Poor growth } \\
\text { Change in colony morphology }\end{array}$ & [7] \\
\hline & & $\begin{array}{l}\text { Microsporum gypseum, } \\
\text { Trichophyton mentagrophytes }\end{array}$ & $\begin{array}{l}\text { MIC: } 0.5-0.4 \%(\mathrm{w} / \mathrm{v}) \\
\text { MFC: } 0.8-0.9 \%(\mathrm{w} / \mathrm{v})\end{array}$ & \multirow[t]{2}{*}[8]{} \\
\hline & & Alternaria spp. & Inhibited spore germination & \\
\hline & & Inonotus obliquus & $\begin{array}{l}\text { Inhibited mycelial growth } \\
\text { Reduced ergosterol production }\end{array}$ & [9] \\
\hline & & $\begin{array}{l}\text { Aspergillus spp. } \\
\text { Fusarium spp. }\end{array}$ & $\begin{array}{c}\text { MIC: } 0.078-0.625 \%(\mathrm{w} / \mathrm{v}) \\
\text { Inhibited conidial germination } \\
\text { May reduce conidial wall permeability to nutrients }\end{array}$ & {$[10]$} \\
\hline & & Eutypa lata & $\begin{array}{l}\text { Inhibited growth, MIC: } 10 \mathrm{mM} \\
\text { Modified structural organization of the mycelium } \\
\text { (complete disorganization of the cell content, nuclear } \\
\text { degeneration) }\end{array}$ & {$[5]$} \\
\hline LCME & NIH & Mucorales spp. & $\begin{array}{l}\text { Reduced hyphal growth, MEC: } 0.625-10 \mathrm{mM} \\
\text { Change in colony morphology } \\
\text { Inhibited sporangiospore germination }\end{array}$ & {$[6]$} \\
\hline NIDC & & Mucorales spp. & Reduced hyphal growth, MEC: $0.625-5 \mathrm{mM}$ & {$[6]$} \\
\hline NILC & & Mucorales spp. & $\begin{array}{l}\text { Reduced hyphal growth, MEC: } 0.625-10 \mathrm{mM} \\
\text { Change in colony morphology }\end{array}$ & {$[6]$} \\
\hline
\end{tabular}

Table 1: Overview of the antifungal activity of the tested cysteine forms and their derivatives.

${ }^{a}$ DC, D-cysteine; LC, L-cysteine; LCME, L-cysteine-methyl ester hydrochloride; NIDC, N-isobutyryl-D-cysteine; NILC, N-isobutyryl-L-cysteine.

bource: http://www.sigmaaldrich.com/

to DC, LC and their derivatives, such as LCME, NIDC, and NILC; and (ii) to investigate the in vitro combinations of the most effective cysteine compound with conventional antifungal agents against clinical Scedosporium isolates. developed for the diagnosis of UTIs. Rapid biochemical dipstick tests are available and currently used as predictors of bacterial UTI, but must often be correlated with other testing and clinical information. In many clinical settings, in fact, it has been proved that the dipstick urinalysis leads to many false positive and negative results when compared with the gold standard culture method, demonstrating the low sensitivity and positive predictive value [10]. The Micro Biological Survey method (MBS) is an alternative method for bacterial counting developed and patented by Roma Tre University [11,12]. It is based on a colorimetric survey performed in mono-use disposable reaction vials in which samples can be inoculated without any preliminary treatment. The analysis can be carried out using a thermostatic optical reader that automatically detects the color change. The ease of use of the MBS method has been evaluated in a previous study on food samples demonstrating the possibility to use it anywhere and without the need of an equipped laboratory and specialized personnel [13]. In previous studies carried out on artificially contaminated urine samples, this method has already been proven to be suitable for the evaluation of the bacterial load and the assessment of the susceptibility to a panel of antibiotics $[14,15]$. The present study has been undertaken to clinically evaluate the performance and operational characteristics of the MBS POCT for the diagnosis and the antibiotic treatment of UTIs. The MBS method was also compared with another biochemical rapid test for UTI diagnosis, namely the urine dipstick assay [16]. 
Citation: Galgóczy L, Homa M, Papp T, Manikandan P, Vágvölgyi C (2016) In Vitro Antifungal Activity of Cysteine Derivatives and their Combinations with Antifungal Agents Against Clinically Relevant Scedosporium species. Int J Clin Med Microbiol 1: 111. doi: https://doi.org/10.15344/2456-4028/2016/111

Page 3 of 5

\section{Materials and Methods}

\section{Strains and culture conditions}

Nine Scedosporium isolates derived from different environmental and clinical sources were involved in this study (Table 2). Prior to the tests, to get the required amount of conidia all the strains were grown on malt extract slants (MEA, Biolab, Hungary) for 2 weeks at $30^{\circ} \mathrm{C}$. Susceptibility tests were performed in RPMI 1640 medium (SigmaAldrich, USA) supplemented with $0.3 \mathrm{~g} / \mathrm{l} \mathrm{L}$-glutamine and buffered to $\mathrm{pH} 7.0$ with $0.165 \mathrm{M}$ 4-morpholinopropanesulfonic acid (SigmaAldrich, USA) and were incubated at $37^{\circ} \mathrm{C}$ for 72 hours.

\section{Results and Discussion}

We evaluated the antifungal effect of two cysteine forms, LC and DC, and their derivatives against nine Scedosporium isolates. The MICs were in the range of $512->1024 \mu \mathrm{g} / \mathrm{ml}$ (Table 2). LCME proved to be most effective among the investigated compounds with a MIC range of $512->1024 \mu \mathrm{g} / \mathrm{ml}$. It demonstrated similar antifungal activity against the tested isolates $(512-1024 \mu \mathrm{g} / \mathrm{ml})$ except for $P$. ellipsoidea CBS 301.79 strain where the MIC was $>1024 \mu \mathrm{g} / \mathrm{ml}$. DC, LC and NIDC were ineffective against the Scedosporium isolates in the investigated concentration range (MIC: $>1024 \mu \mathrm{g} / \mathrm{ml}$ ). While NILC proved to be active against one strain only, S. aurantiacum CBS

\begin{tabular}{|l|l|l|l|l|l|}
\hline \multirow{2}{*}{ Species (Strain numbera) } & Source & \multicolumn{3}{l|}{ Mean MICs of cysteine and its derivativesb $(\mu \mathrm{g} / \mathrm{ml})$} \\
\cline { 3 - 6 } & & DC & LC & LCME & NIDC \\
\hline S. aurantiacum (CBS 116910) & Wound exudate/Spain & $>1024$ & $>1024$ & 512 & $>1024$ \\
\hline S. aurantiacum (CBS 136046) & Human lung/Australia & $>1024$ & $>1024$ & 1024 & 1024 \\
\hline S. aurantiacum (CBS 136047) & Soil/Australia & $>1024$ & $>1024$ & 1024 & $>1024$ \\
\hline S. aurantiacum (CBS 136049) & Soil/Austria & $>1024$ & $>1024$ & 1024 & $>1024$ \\
\hline S. boydii (CBS 117410) & Soil/Spain & $>1024$ & $>1024$ & 1024 & $>1024$ \\
\hline S. boydii (CBS 117432) & Sputum/France & $>1024$ & $>1024$ & 1024 & $>1024$ \\
\hline S. boydii (CBS 120157) & Human lung/France & $>1024$ & $>1024$ & 1024 & $>1024$ \\
\hline P. angusta (CBS 254.72) & Sewage/USA & $>1024$ & $>1024$ & 1024 & $>1024$ \\
\hline P. ellipsoidea (CBS 301.79) & Dung/The Netherlands & $>1024$ & $>1024$ & $>1024$ & $>1024$ \\
\hline MIC range & & $>1024$ & $>1024$ & $512->1024$ & $>1024$ \\
\hline
\end{tabular}

Table 2 The antifungal activity of cysteine and its derivatives against Scedosporium isolates.

${ }^{a}$ CBS, Centraalbureau voor Schimmelcultures, Utrecht, The Netherlands.

${ }^{b}$ DC, D-cysteine; LC, L-cysteine; LCME, L-cysteine-methyl ester hydrochloride; NIDC, N-isobutyryl-D-cysteine; NILC, N-isobutyryl-L-cysteine.

\section{Microdilution tests}

The susceptibility of Scedosporium isolates to cysteine forms and their derivatives were determined following the slightly modified instructions of the Clinical and Laboratory Standards Institute's M38-A2 broth microdilution method [13]. Minimum inhibitory concentration (MIC) was defined as the lowest concentration of a drug which was required for the total growth inhibition of a certain isolate after 72 hours-long incubation. Modifications connected to stock solution and inoculum preparation were detailed previously [14]. We evaluated the antifungal effect of five compounds: DC, LC, LCME, NIDC, and NILC (Sigma-Aldrich, USA). The final drug concentrations in the tests ranged from 64 to $1024 \mu \mathrm{g} / \mathrm{ml}$.

Drug interactions between LCME and conventional antifungal agents (i.e., amphotericin B, AMB; caspofungin, CSP; terbinafine, TRB; and voriconazole, VRC) were investigated against the four clinical isolates using the checkerboard microdilution method [15]. The final LCME concentrations ranged from 64 to $2048 \mu \mathrm{g} / \mathrm{ml}$. The final concentrations of the antifungal drugs were between 0.25 and $128 \mu \mathrm{g} / \mathrm{ml}$. Fractional inhibitory concentration indexes (FICIs) were calculated to demonstrate the type of interaction between the paired combinations of compounds [16]. For these calculations, the MICs of antifungals have been already determined in a recent study of our research group [14]. Synergism was defined as FICI $\leq 0.5$, indifference as $0.5<\mathrm{FICI} \leq 4$ and antagonism was defined when FICI $>4$ [17].

Both single and combination tests were performed in three replicates.
116910 (MIC: $1024 \mu \mathrm{g} / \mathrm{ml}$ ). Differences between the susceptibilities of environmental and clinical isolates to the investigated compounds were not observed.

The antifungal activity of cysteine and its derivatives against Scedosporium species has not been investigated yet. The MICs observed in this study are comparable to previously reported data against other fungal species. The in vitro inhibitory effect of DC was proved previously against Eutypa lata and different Mucorales species. It caused reduced hyphal growth in a concentration of $0.625-10 \mathrm{mM}$ $(\sim 75-1200 \mu \mathrm{g} / \mathrm{ml})[5,6]$. The antifungal effect of LC was reported against different filamentous fungi: dermatophytes, Alternaria spp., Aspergillus spp., Eutypa lata, Fusarium spp., and Inonotus obliquus [511]. Our previous publication showed the antifungal effect of LCME, NIDC, and NILC against Mucorales species: the MEC values were between 0.625 and $10 \mathrm{mM}(\sim 100-1700 \mu \mathrm{g} / \mathrm{ml}$ at LCME; $\sim 60-1000$ $\mu \mathrm{g} / \mathrm{ml}$ at NIDC and NILC) [6]. Beside the reduced hyphal growth, LCME inhibited the germination of sporangiospores, while the presence of NILC in the culture media led to changes in the colony morphology [6]. The mode of action of cysteine and their derivatives has not been clarified yet. Kahlos and Tikka [10] observed that LC reduced the ergosterol production of Inonotus ubliquus. Octave et al. [5] reported the complete structural disorganization of the mycelia of Eutypa lata. According to Daigle and Cotty [9] the mercaptan and amino groups of LC were essential to the inhibition of conidial germination.

In the combination tests, the in vitro interactions between the most effective non-antifungal agent, LCME and four conventional 
Citation: Galgóczy L, Homa M, Papp T, Manikandan P, Vágvölgyi C (2016) In Vitro Antifungal Activity of Cysteine Derivatives and their Combinations with Antifungal Agents Against Clinically Relevant Scedosporium species. Int J Clin Med Microbiol 1: 111. doi: https://doi.org/10.15344/2456-4028/2016/111

Page 4 of 5

antifungal drugs were evaluated. These results are summarized in Table 3. We revealed no antagonistic interaction for LCME with any of the antifungal drugs. Between LCME and AMB, and LCME and TRB only synergistic interactions were observed (FICI ${ }_{\mathrm{LCME}+\mathrm{AMB}}: 0.06$ $0.13, \mathrm{FICI}_{\mathrm{LCME}+\mathrm{TRB}}: 0.09-0.16$ ). For $S$. aurantiacum CBS 116910 strain, the drug interaction between LCME and CSP and between LCME and VRC proved to be indifferent. However, we found synergistic interaction in all other cases between these compounds $\left(\mathrm{FICI}_{\mathrm{LCME}+\mathrm{CSP}}\right.$ : 0.13 - 0.63, FICI ${ }_{\mathrm{LCME}+\mathrm{VRC}}: 0.13$ - 1.00) (Table 3). Previously, we investigated the in vitro combinations of another L-cysteine derivative, $\mathrm{N}$-acetyl-cysteine with AMB, CSP, TRB, and VRC [12]. Similarly, synergistic interactions were revealed predominantly between the investigated agents and antagonistic interactions were not registered.

\section{Conclusions}

This is the first study investigating the activity of cysteine derivatives against Scedosporium species and the first report of the in vitro antifungal effect of LCME and its synergistic interactions with conventional antifungal agents against Scedosporium isolates. According to our results, LCME alone or in combination with other drugs might be useful for the treatment of human Scedosporium infections. However, further studies are required to investigate the antifungal mechanism of LCME and its pharmacokinetic and pharmacodynamic properties.

\section{Competing Interests}

The authors declare that they have no competing interests.

\begin{tabular}{|c|c|c|c|c|c|c|}
\hline Isolate $^{\mathrm{a}}$ & Mean MIC $(\mu \mathrm{g} / \mathrm{ml})^{b}$ & $\mathrm{FICI}^{c}$ & Interaction $^{d}$ & & & \\
\hline & LCME $_{\text {alone }}$ & LCME $_{\text {comb }}$ & $\mathrm{AMB}_{\text {alone }}{ }^{*}$ & $\mathrm{AMB}_{\text {comb }}$ & & \\
\hline S. aurantiacum (CBS 116910) & 512 & 64 & 128 & 1 & 0.13 & S \\
\hline S. aurantiacum (CBS 136046) & 1024 & 64 & 128 & 4 & 0.09 & S \\
\hline S. boydii (CBS 117432) & 1024 & 64 & 128 & 0.25 & 0.06 & S \\
\hline \multirow[t]{2}{*}{ S. boydii (CBS 120157) } & 1024 & 64 & 64 & 2 & 0.09 & $S$ \\
\hline & $\mathrm{LCME}_{\text {alone }}$ & $\mathrm{LCME}_{\text {comb }}$ & $\mathrm{CSP}_{\text {alone }}{ }^{*}$ & $\mathrm{CSP}_{\text {comb }}$ & & \\
\hline S. aurantiacum (CBS 116910) & 512 & 64 & 32 & 16 & 0.63 & $\mathrm{NI}$ \\
\hline S. aurantiacum (CBS 136046) & 1024 & 128 & 64 & 0.125 & 0.13 & $S$ \\
\hline S. boydii (CBS 117432) & 1024 & 64 & 64 & 8 & 0.19 & $S$ \\
\hline \multirow[t]{2}{*}{ S. boydii (CBS 120157) } & 1024 & 128 & 32 & 0.125 & 0.13 & S \\
\hline & LCME $_{\text {alone }}$ & LCME $_{\text {comb }}$ & $\mathrm{TRB}_{\text {alone }}{ }^{*}$ & $\mathrm{TRB}_{\text {comb }}$ & & \\
\hline S. aurantiacum (CBS 116910) & 512 & 64 & 128 & 4 & 0.16 & $S$ \\
\hline S. aurantiacum (CBS 136046) & 1024 & 64 & 128 & 4 & 0.09 & $S$ \\
\hline S. boydii (CBS 117432) & 1024 & 64 & 128 & 4 & 0.09 & S \\
\hline \multirow[t]{2}{*}{ S. boydii (CBS 120157) } & 1024 & 64 & 128 & 8 & 0.13 & S \\
\hline & LCMEalone & LCME $_{\text {comb }}$ & $\mathrm{VRC}_{\text {alone }}{ }^{*}$ & $\mathrm{VRC}_{\text {comb }}$ & & \\
\hline S. aurantiacum (CBS 116910) & 512 & 256 & 8 & 4 & 1.00 & $\mathrm{NI}$ \\
\hline S. aurantiacum (CBS 136046) & 1024 & 128 & 32 & 0.125 & 0.13 & S \\
\hline S. boydii (CBS 117432) & 1024 & 64 & 16 & 4 & 0.31 & S \\
\hline S. boydii (CBS 120157) & 1024 & 64 & 16 & 1 & 0.13 & S \\
\hline
\end{tabular}

Table 3: The combination test results of LCME and conventional antifungal drugs against clinical Scedosporium isolates.

${ }^{a} \mathrm{CBS}$, Centraalbureau voor Schimmelcultures, Utrecht, The Netherlands.

${ }^{b} \mathrm{LCME}_{\text {alone }}, \mathrm{AMB}_{\text {alone }}, \mathrm{CSP}_{\text {alone }}, \mathrm{TRB}_{\text {alone }}$ and $\mathrm{VRC}_{\text {alone }}$, mean MICs of L-cysteine methyl ester hydrochloride, amphotericin B, caspofungin, terbinafine and voriconazole, respectively, when applied alone; $\mathrm{LCME}_{\text {comb }}, \mathrm{AMB}_{\text {comb }}, \mathrm{CSP}_{\text {comb }}, \mathrm{TRB}_{\text {comb }}$ and $\mathrm{VRC}_{\text {comb }}$, mean MICs of L-cysteine methyl ester hydrochloride, amphotericin B, caspofungin, terbinafine and voriconazole, respectively, when applied in combination.

${ }^{c}$ FICI, fractional inhibitory concentration index value

NI, no interaction $(0.5<\mathrm{FICI} \leq 4)$; S, synergism $(\mathrm{FICI} \leq 0.5)[17]$

${ }^{\star}$ The MICs of AMB, CSP, TRB and VRC were determined in a previous study of our research group [14].

When used alone, the MIC range of LCME were 512 - $1024 \mu \mathrm{g} /$ $\mathrm{ml}$, but in combination with AMB, CSP, TRB, and VRC, these values reduced to $64 \mu \mathrm{g} / \mathrm{ml}, 64-128 \mu \mathrm{g} / \mathrm{ml}, 64 \mu \mathrm{g} / \mathrm{ml}$, and $64-256 \mu \mathrm{g} / \mathrm{ml}$, respectively. Compared to the single use, the MICs of antifungal agents were also decreased in the combination tests. The previously determined MIC ranges of AMB, CSP, TRB, and VRC were $8-128$ $\mu \mathrm{g} / \mathrm{ml}, 32-64 \mu \mathrm{g} / \mathrm{ml}, 128 \mu \mathrm{g} / \mathrm{ml}$ and $8-64 \mu \mathrm{g} / \mathrm{ml}$, respectively [14]. In combination with LCME, the MIC ranges of AMB, CSP, TRB, and VRC were decreased to 0.25 - $4 \mu \mathrm{g} / \mathrm{ml}, 0.125$ - $16 \mu \mathrm{g} / \mathrm{ml}, 4-8 \mu \mathrm{g} / \mathrm{ml}$, and $0.125-4 \mu \mathrm{g} / \mathrm{ml}$, respectively (Table 3 ). Compared to the single use, the MIC values of antifungal drugs in the combination tests could be decreased to their achievable therapeutic plasma concentrations in several cases [18-21].

\section{Author Contributions}

László Galgóczy, Tamás Papp, Palanisamy Manikandan, and Csaba Vágvölgyi took part in the experimental design, the evaluation of the results and the final preparation of the manuscript. The single and combination tests for the fungal isolates were carried out by László Galgóczy and Mónika Homa.

\section{Acknowledgements}

L.G. holds a Lise Meitner-Position (M 1776-B20) from the Austrian Science Fund (FWF). T.P. is a grantee of J. Bolyai Scientific Scholarship of the Hungarian Academy of Sciences. The study was supported by the European Union co-financed by the European Social 
Citation: Galgóczy L, Homa M, Papp T, Manikandan P, Vágvölgyi C (2016) In Vitro Antifungal Activity of Cysteine Derivatives and their Combinations with Antifungal Agents Against Clinically Relevant Scedosporium species. Int J Clin Med Microbiol 1: 111. doi: https://doi.org/10.15344/2456-4028/2016/111

Page 5 of 5

Fund (TÁMOP-4.2.2.B-15/1/KONV-2015-0006). This work was also funded by the grant LP2016-8/2016.

\section{References}

1. Cortez KJ, Roilides E, Quiroz-Telles F, Meletiadis J, Antachopoulos C, et al. (2008) Infections caused by Scedosporium spp. Clin Microbiol Rev 21: 157-197.

2. Tortorano AM, Richardson M, Roilides E, van Diepeningen A, Caira M, et al (2014) ESCMID and ECMM joint guidelines on diagnosis and management of hyalohyphomycosis: Fusarium spp., Scedosporium spp. and others. Clin Microbiol Infect 20: 27-46.

3. Alastruey-Izquierdo A, Mellado E, Peláez T, Pemán J, Zapico S, et al. (2013) Population-based survey of filamentous fungi and antifungal resistance in Spain (FILPOP Study). Antimicrob Agents Chemother 57: 3380-3387.

4. Guarro J, Kantarcioglu AS, Horré R, Rodriguez-Tudela JL, Cuenca Estrella $\mathrm{M}$, et al. (2006) Scedosporium apiospermum: changing clinical spectrum of a therapy-refractory opportunist. Med Mycol 44: 295-327.

5. Octave S, Amborabé BE, Luini E, Ferreira T, Fleurat-Lessard P, Roblin G (2005) Antifungal effects of cysteine towards Eutypa lata, a pathogen of vineyards. Plant Physiol Biochem 43: 1006-1013.

6. Galgóczy L, Kovács L, Krizsán K, Papp T, Vágvölgyi C (2009) Inhibitory effects of cysteine and cysteine derivatives on germination of sporangiospores and hyphal growth of different Zygomycetes. Mycopathologia 168: 125-134.

7. Nguyen NT, Galgóczy J, Novák EK (1981) Morphogenetic effect of L-cysteine on dermatophytes. Acta Microbiol Acad Sci Hung 28: 347-357.

8. Pandey DK, Chandra H, Tripathi NN, Dixit SN (1984) Antimycotic activity of some amino acids against dermatophytes. Arzneimittelforschung 34: 554 556

9. Daigle DJ, Cotty PJ (1991) The influence of cysteine, cysteine analogs and other amino acids on spore germination of Alternaria species. Can $J$ Bot 69: 2353-2356.

10. Kahlos K, Tikka VH (1994) Antifungal activity of cysteine, its effect on C-21 oxygenated lanosterol derivatives and other lipids in Inonotus obliquus, in vitro. Appl Microbiol Biotechnol 42: 385-390.

11. De Lucca AJ, Walsh TJ, Daigle DJ (1996) N-acetylcysteine inhibits germination of conidia and growth of Aspergillus spp. and Fusarium spp. Antimicrob Agents Chemother 40: 1274-1276.

12. Homa M, Galgóczy L, Tóth E, Virágh M, Chandrasekaran M, et al. (2016) In vitro susceptibility of Scedosporium isolates to $\mathrm{N}$-acetyl-L-cysteine alone and in combination with conventional antifungal agents. Med Mycol.

13. CLSI (2008) Reference method for broth dilution antifungal susceptibility testing of filamentous fungi. In: Approved standard CLSI document M38-A2. Wayne: Clinical and Laboratory Standards Institute, USA.

14. Homa M, Galgóczy L, Tóth E, Tóth L, Papp T, Chandrasekaran M Kadaikunnan S, Alharbi NS, Vágvölgyi C (2015) In vitro antifungal activity of antipsychotic drugs and their combinations with conventional antifungals against Scedosporium and Pseudallescheria isolates. Med Mycol 53: 890895.

15. Eliopoulos GM, Moellering RC (1996) Antimicrobial combinations. In: Lorian $\vee(E d)$ Antibiotics in Laboratory Medicine (4th edn), Baltimore: The Williams and Wilkins Co., USA, 330-396 pp.

16. Johnson MD, MacDougall C, Ostrosky-Zeichner L, Perfect JR, Rex JH (2004) Combination antifungal therapy. Antimicrob Agents Chemother 48: 693-715.

17. Odds FC (2003) Synergy, antagonism, and what the chequerboard puts between them. J Antimicrob Chemother 52: 1.

18. Bekersky I, Fielding RM, Dressler DE, Lee JW, Buell DN, Walsh TJ (2002) Plasma protein binding of amphotericin B and pharmacokinetics of bound versus unbound amphotericin $B$ after administration of intravenous liposomal amphotericin B (AmBisome) and amphotericin B deoxycholate. Antimicrob Agents Chemother 46: 834-840.

19. Nguyen TH, Hoppe-Tichy T, Geiss HK, Rastall AC, Swoboda S, et al. (2007) Factors influencing caspofungin plasma concentrations in patients of a surgical intensive care unit. J Antimicrob Chemother 60: 100-106.

20. Lewis RE (2008) What is the "therapeutic range" for voriconazole? Clin Infect Dis 46: 212-214
21. Dolton MJ, Perera V, Pont LG, McLachlan AJ (2014) Terbinafine in combination with other antifungal agents for treatment of resistant or refractory mycoses: investigating optimal dosing regimens using a physiologically based pharmacokinetic model. Antimicrob Agents Chemother 58: 48-54. 